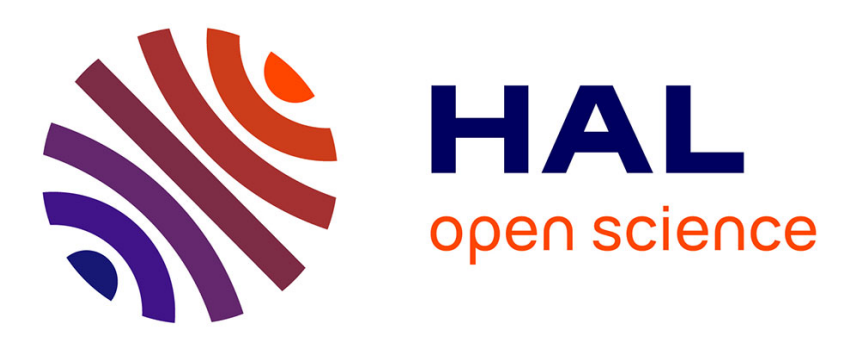

\title{
In-Vitro and Numerical Investigations of the Influence of a Vocal-Tract Resonance on Lip Auto-Oscillations in Trombone Performance
}

\author{
V Fréour, N Lopes, Thomas Hélie, R Caussé, G.P. Scavone
}

\section{- To cite this version:}

V Fréour, N Lopes, Thomas Hélie, R Caussé, G.P. Scavone. In-Vitro and Numerical Investigations of the Influence of a Vocal-Tract Resonance on Lip Auto-Oscillations in Trombone Performance. Acta Acustica united with Acustica, 2015, 101 (2), pp.256-269. 10.3813/AAA.918824 . hal-01277959

\section{HAL Id: hal-01277959 \\ https://hal.science/hal-01277959}

Submitted on 24 Feb 2016

HAL is a multi-disciplinary open access archive for the deposit and dissemination of scientific research documents, whether they are published or not. The documents may come from teaching and research institutions in France or abroad, or from public or private research centers.
L'archive ouverte pluridisciplinaire HAL, est destinée au dépôt et à la diffusion de documents scientifiques de niveau recherche, publiés ou non, émanant des établissements d'enseignement et de recherche français ou étrangers, des laboratoires publics ou privés. 


\title{
In-vitro and numerical investigations of the influence of a vocal-tract resonance on lip auto-oscillations in trombone performance
}

\author{
V. Fréour ${ }^{1)}$, N. Lopes ${ }^{2)}$, T. Hélie ${ }^{2)}$, R. Caussé2), G.P. Scavone ${ }^{1)}$ \\ 1) Computational Acoustic Modeling Laboratory, Centre for Interdisciplinary Research in Music \\ Media and Technology (CIRMMT), Schulich School of Music, McGill University, Montréal, \\ Québec H3A 1E3, Canada \\ vincent.freour@mail.mcgill.ca \\ 2) IRCAM-CNRS UMR 9912 Sciences et Technologies de la Musique et du Son, Université \\ Pierre et Marie Curie, 1 place Igor Stravinsky, 75004 Paris, France
}

November 3, 2014 


\begin{abstract}
Controlling the acoustic impedance of the upstream airways in brass instrument performance may be an important factor influencing the efficiency of the sound production process. Because of the complex characteristics of the lip-valve oscillator, the conditions under which a vocal-tract resonance may be favorable to the sustain of lip auto-oscillations are not easy to determine. In order to investigate this aspect of brass performance, an experimental method based on an active control approach is applied to an artificial trombone player system in order to simulate the influence of a vocal-tract resonance at the playing frequency. By varying the amplitude and phase characteristics of this upstream impedance load, we investigate the acoustical influence of this resonance on lip vibrations and on the acoustic pressure generated in the instrument. The observations reveal that variations of the phase difference between the downstream and upstream impedance induce significant variations of the playing frequency. An optimal phase tuning point characterized by a maximum of downstream acoustic pressure at the input of the instrument, and uncorrelated to a maximum of downstream input impedance, is identified. These experimental results are compared with numerical simulations, both of which produce similar findings. The optimal tuning point appears to be partly related to the displacement of the playing frequency close to a mechanical resonance of the lips. This induces a greater "efficiency" of the lip-valve system, hence maximizing the acoustic flow generated into the instrument while other control parameters (quasi-static mouth pressure, lip tension) are maintained constant. In addition to an exploration of acoustical influence of the vocal tract, this experimental method hence offers promising perspectives for the study of artificial lips under playing conditions.
\end{abstract}

PACS numbers: 43.75.Fg, 43.75.St, 43.75.Yy 


\section{Introduction}

The study of the acoustical influence of upstream airways in brass instrument performance has been the object of growing interest in the last years $[1,2,3,4]$. In trombone performance, significant vocal-tract influence in the high register of the instrument was observed by measuring the ratio of the acoustic pressures upstream and downstream from the lips [4]. Furthermore, in the same study, the characteristics of the upstream and downstream impedances at the playing frequency could be derived by monitoring the phase of lip oscillations, and an early categorization of the upstream tuning strategy proposed during arpeggios. These observations highlight the potential importance of phase tuning of the vocal-tract impedance on the behaviour of the lips. In order to clarify the modalities of the acoustical interaction between the vocal-tract and the vibrating lips, it hence becomes necessary to work under more controlled conditions. Consequently, the aim of this study is to investigate the influence of an upstream coupling on an artificial player system and numerically on different physical models of the lips, in order to inform the analysis of experimental results obtained on musicians.

Several artificial player systems have been used in music acoustic research over the last decades. These "blowing machines" have been developed with different degrees of complexity and applied to the excitation of woodwind instruments $[5,6,7,8,9]$, as well as to brass instruments where the vibrating lips are typically modeled by a pair of latex tubes filled with a liquid or fluid element $[10,11,12,13,14]$. These systems constitute a basic model of a wind player by combining replicas of the main components involved in the mechanism of sound production. Moreover, these artificial players provide substantial advantages over human subjects: 1) control parameters such as the embouchure and the quasi-static mouth pressure can be controlled and maintained constant over a long period of time, 2) a number of measurement sensors may be embedded within the system without disturbing the sound production process, 3) some of these systems are now automatized and allow for precise feedback control and monitoring of various physical parameters, 4) such a system is by nature inexpressive and neither susceptible to any conscious or unconscious playing conditioning as it is the case for a musician.

This experimental setup thus potentially enables variations of upstream coupling characteristics independently from embouchure adjustments, which is obviously not possible in human subjects. The influence

of different upstream coupling conditions on the behaviour of the lips may therefore be investigated in order to derive the nature of an optimal vocal-tract tuning. 
Most recent attempts to incorporate a vocal-tract resonator to an artificial player system were performed on the clarinet $[15,16]$ and on the didjeridu and trombone [1]. On the dijeridu and trombone, a system enabled the characteristics of an upstream resonance to be varied (low or high tongue position roughly corresponding respectively to vowels [æ] and [I]) by changing the degree of constriction just upstream from the lips, while maintaining a fixed reed setting. On both instruments, vocal-tract configuration had an effect on intonation as well as on transitions between two registers. On the clarinet, two vocal-tract configurations corresponding to vowels "aw" and "ee" were realized by assembling plastic rings of different cross-section areas. Although this method allows for simulation of more realistic vocal-tract shapes, the low versatility imposes a reduced number of configurations and makes time-varying manipulation of the vocal-tract impossible. Moreover, the discontinuities at ring junctions may produce aeroacoustical effects interfering with the expected acoustical influence of the vocal-tract.

For this reason, we chose to apply another method based on the injection of an acoustic flow in the mouth cavity of the artificial player, and inspired from previous work by Chen and Weinreich [17]. By properly adjusting the phase and amplitude of the injected signal, we aim to simulate different vocal-tract impedance conditions at the playing frequency using an active control method. This approach was also recently adopted for the study of flute-like instruments [18].

Physical modeling has been extensively applied to synthesis of wind instrument sounds. Based on the simulation of the physical mechanisms underlying sound production, this approach can also be employed as a numerical benchmark for the study of the physical behaviour of the instrument and player's control. In brass instruments, particular efforts have been devoted to the development of realistic models of the lip-valve excitation mechanism. Physical representations of lip-reed valves commonly consider the lips as identical and placed symmetrically against the mouthpiece, then reducing the problem to the modeling of one lip.

Numerical investigations including the effect of vocal-tract resonances in wind instruments have been performed in different ways. Sommerfeldt and Strong [19] proposed a detailed time-domain simulation of a player-clarinet system involving a six-segment cylindrical tube representation of the player's vocal-tract. Various vocal-tract configurations were explored, some of them producing noticeable changes in the radiated sound spectrum. Fritz et al. proposed an analytical formulation and frequency-domain simulations of the frequency shift induced by a vocal-tract resonance in clarinets [15]. That work showed some potential effect on the playing frequency when an upstream resonance is aligned with a downstream impedance resonance, though these effects were not clearly described. Scavone investigated the effect of a single resonance on the 
sound produced by a simple saxophone physical model using the digital waveguide scheme [20]. Simulated effects of the upstream resonance on the spectral content of the mouthpiece pressure were found to be consistent with real playing situations. Furthermore, Guillemain proposed a refined representation of upstream airways for the simulation of transients on a clarinet model [21]. In the latter study, the player's vocal-tract was represented by a cylindrical tube including frequency-dependent losses, which aimed at simulating the effect of the first impedance peak of the upstream system. Contrary to previous approaches, the quasi-static pressure was supplied at the entrance of the upstream bore rather than at the reed level, providing a more coherent representation of the physics of the system. Most recently, time-domain simulations including the impedance of the vocal-tract calculated by a transfer matrix method from vocal-tract visualization were performed by Kaburagi et al. [2]. Different upstream conditions were characterized from MRI imaging of a trumpet player's vocal-tract and tested on the numerical model of the lips proposed by Adachi and Sato [22]. Vocal-tract configuration especially appeared to influence the oscillation threshold pressure, as well as the transition between adjacent modes.

In this paper, we propose a different approach to what has been previously presented. Our method attempts to replicate the active control method applied to the artificial player system. Therefore, by injecting a disturbing signal of frequency $f_{0}$ with controllable amplitude and phase (where $f_{0}$ denotes the varying playing frequency), we aim to simulate varying characteristics of an upstream resonance centered around the playing frequency. We apply this strategy to a single degree of freedom outward striking model, as well as to the two-dimensional model proposed by Adachi and Sato [22] as it seems to produce a close representation of the behaviour of human lips.

This paper is organized as follows: in Section 2, the active control method applied to the in-vitro study is described, and the results from experiments are presented. In Sections 3 and 4, the numerical scheme is introduced for two types of lip models and results from simulations discussed in light of a linear theory of oscillation. Finally, Sec. 5 summarizes the contributions and concludes in the context of the analysis of trombone performance. 


\section{In-vitro investigation}

\subsection{Artificial player system}

This study was performed using the artificial player system developed at IRCAM [13]. This system includes a mouth cavity of cylindrical shape and two cylindrical artificial lips made of latex and filled with water. The system allows control of the following parameters:

- Air flow injected in the mouth cavity via an electrical servo valve.

- Volume of water in each lip controlled independently by two actuators.

- Longitudinal position of the artificial mouth mounted on a translator, relative to the mouthpiece position.

These controls regulate on a number of physical variables of the system. Various sensors embedded into the artificial player allow access to the following variables:

- Water pressure in the lips, providing an image of the tension of each lip.

- Force applied by the lips on the mouthpiece.

- Quasi-static mouth pressure.

- Lip opening area measured using a light transmission method as described in previous studies [23, 24].

The communication between the control PC, the actuators, and the different sensors is performed through a dSpace ${ }^{\odot}$ system including an input/output interface working at $1 \mathrm{kHz}$ sampling frequency. This module also includes a digital signal processor that is programmed using a dSpace ${ }^{\circledR}$ software system interfacing with a Matlab-Simulink-RTW ${ }^{\odot}$ environment [14]. This system therefore enables continuous streams of commands and data from the sensors. Feedback control on the actuator commands can be performed, allowing stable settings to be maintained during experiments.

By construction, the mouth cavity presents some acoustical characteristics potentially responsible for an inherent acoustical coupling with the lips. This upstream coupling may occur at frequencies where the amplitude of the upstream acoustic pressure is significant enough compared with the downstream pressure. 
Alternatively, by comparing the amplitude of the input impedance of the trombone and mouth cavity measured experimentally, we may assess the "default" acoustical influence of the mouth cavity.

The input impedance of the valve trombone used for experiments was measured in the valve position 000 (no valve pressed). Analogously, the input impedance of the mouth cavity was measured at the lip orifice. This was performed by removing the artificial lips from their enclosure and adjusting the impedance probe against the mouth cavity, at the location where the lips are normally mounted. An upstream resonance is observed around $400 \mathrm{~Hz}$. The amplitude of this impedance peak being less than half the amplitude of

the closest downstream resonance, we consider that the default acoustical coupling with the mouth cavity is overall negligible in comparison with the acoustical feedback from the downstream air-column. We may further infer that, away from this upstream resonance, the acoustic pressure originating from this upstream feedback can be ignored in our active control approach and therefore assume that the mouth cavity behaves like an infinite volume reservoir of null input impedance $\left(Z_{u} \simeq 0\right)$. Close to $400 \mathrm{~Hz}$, a small upstream feedback may occur and a stronger external acoustic signal may be needed during active control to counterbalance the inherent effect of the mouth cavity. Another resonance was also observed around $1500 \mathrm{~Hz}$, although it is not expected to interfere acoustically with the lips at $f_{0}$ in the frequency region of interest.

\subsection{Simulation of a vocal-tract resonance using active control}

Under the assumption of a limited acoustical feedback from the mouth cavity, a method based on the feedback control of the acoustic pressure created in the mouth cavity is developed. Assuming continuity of the volume flow at the reed junction, Eq. 1 can be derived in the Fourier domain:

$$
\frac{Z_{u}(\omega)}{Z_{d}(\omega)}=-\frac{P_{u}(\omega)}{P_{d}(\omega)}
$$

where $Z_{u}$ and $Z_{d}$ denote the upstream and downstream impedances respectively, and $P_{u}$ and $P_{d}$ the acoustic pressure upstream and downstream from the lips. Using this relation, different conditions of acoustical coupling with the upstream system relative to the downstream system can be simulated at $f_{0}$. This is performed by adjusting the amplitude and phase of the pressure at the input of the mouth cavity $P_{u}$ relative to the pressure at the input of the downstream air-column $P_{d}$. This approach hence requires the ability to estimate the amplitude and phase of $P_{d}$ and $P_{u}$ at $f_{0}$ through short window frames in order to allow for real-time adjustments of the amplitude and phase ratio between $Z_{u}$ and $Z_{d}$. Note that this approach is not necessarily 


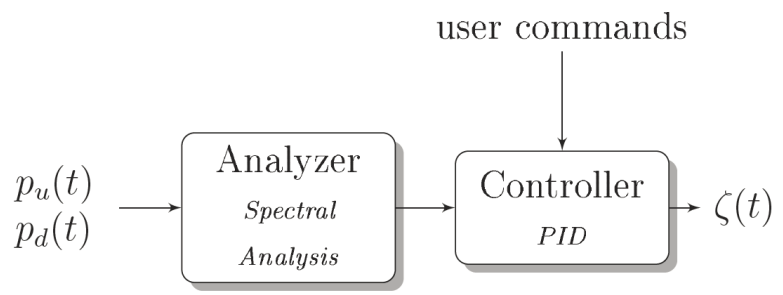

Figure 1: Active control method. The input signals are $p_{u}(t)$ and $p_{d}(t)$ measured by the upstream and downstream microphones, and the output signal is the electrical voltage at the terminals of the compression driver $\zeta(t)$. The spectral analysis and PID control are implemented using the visual programming language Max/MSP.

limited to a control at $f_{0}$ alone. Indeed, it is theoretically possible to impose an upstream feedback with several harmonics of the fundamental frequency, but this was not tested in this study.

The general principle of our active control approach is illustrated in Fig. 1. The input signals are the upstream and downstream acoustic pressures measured on the upstream and downstream sides of the lips respectively. The output signal is the voltage $\zeta(t)$ at the terminals of a compression driver generating an acoustic flow into the mouth cavity and calculated from Eq. 2. The analyzer allows for real-time estimation of the amplitude and phase of $P_{u}$ and $P_{d}$ at the fundamental frequency $f_{0}$. A PID (proportional integral derivative) controller enables adjustment of $\zeta(t)$ according to the output of the analyzer and input commands from the user. The analyzer and controller blocks are detailed in the following paragraphs.

The spectral analyzer is implemented in the programming language Max/MSP. This programming environment allows for the implementation of real-time digital signal processing tools operating at a high sampling rate suitable for audio applications. One issue concerns the pseudo real-time estimation of the amplitude and phase of $p_{u}(t)$ and $p_{d}(t)$ at the fundamental frequency $f_{0}$. The solution proposed is illustrated in Fig. 2. This method uses 2048-sample Fast Fourier transforms applied to modulated versions of $p_{u}(t)$ and $p_{d}(t)$. Calling $N$ the FFT size $(N=2048$ samples), the frequency step of analysis can then be calculated: $\eta=f_{s} / N$. The extraction of the fundamental frequency is achieved using the Max/MSP external fiddle which computes the value of $f_{0}$ over 2048-sample windows from the downstream pressure signal $p_{d}(t)$ [25]. In order to evaluate the phase and amplitude ratio of $P_{u}$ to $P_{d}$ at $f_{0}$, the spectral resolution would need to be increased around the fundamental frequency peak. This could be achieved by increasing the analysis window size N. Alternatively, the frequency sampling could be increased by applying zero padding to a smaller analysis window. The first approach is not compatible with our requirement of achieving a fast evaluation 


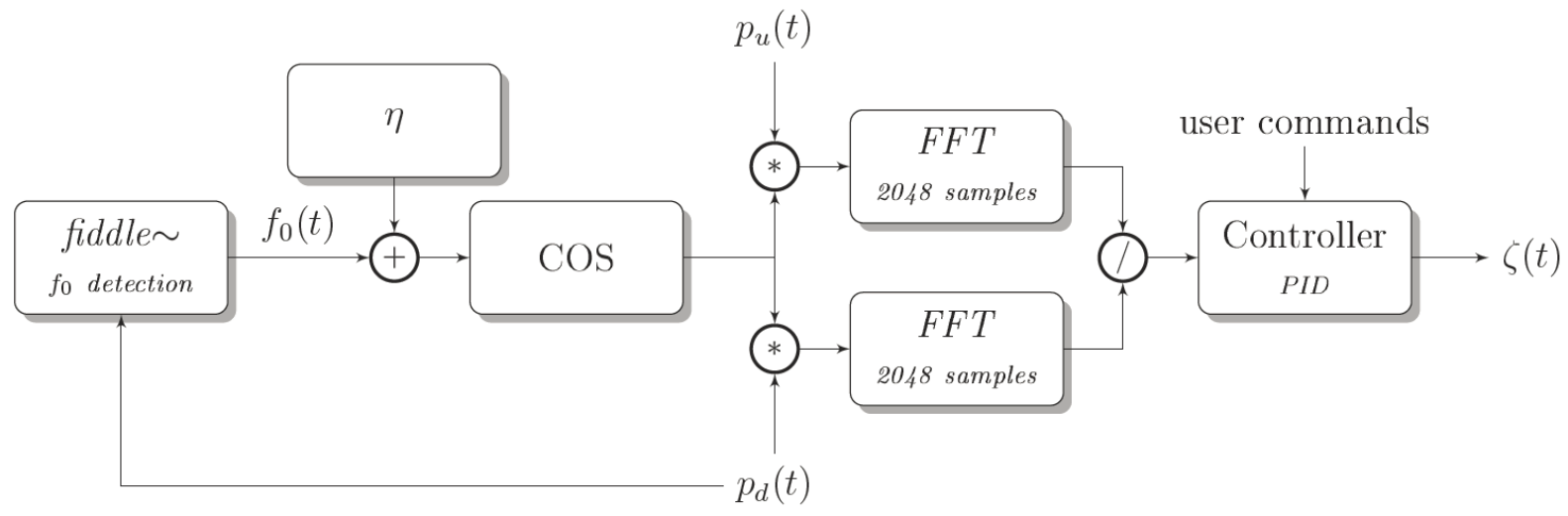

Figure 2: Diagram description of the analyzer implemented in Max/MSP.

and is constrained by the boundary value imposed by Max/MSP (4096 samples in Max/MSP 6.1.3 32-bit). The second approach would slow down the process and is not permitted in Max/MSP.

To solve this issue, we adopt an alternative strategy: at each time step, $p_{u}(t)$ and $p_{d}(t)$ are both modulated by a cosine signal of frequency $f_{0}+\eta$. The modulated signals are then sent to the input of an $f f t \sim$ object of window-size 2048 samples and 0\% overlap. The amplitude and phase are extracted from the real and imaginary outputs of the $f f t \sim$ object and stored in two buffers. The modulation applied before the FFT hence results in a displacement of the fundamental frequency peak to the second frequency bin of the computed spectrum (equivalent to ring modulation). The amplitude and phase values at the second bin are then read from the buffers for each computed FFT, at a rate imposed by the $f f t \sim$ object.

Adopting a discrete-time notation and denoting $T_{s}$ the sampling period, the complex representation of the output parameter $\zeta[n]$ controlling the electrical voltage at the terminals of the compression driver is given by Eq. 2:

$$
\underline{\zeta}[n]=A[n] \cdot \exp j\left(2 \pi T_{s} \sum_{k=0}^{n} f_{0}[k]+\Phi[n]\right) .
$$

Parameters $A[n]$ and $\phi[n]$ of the output signal $\zeta[n]$ are calculated from Eqs. 6 and 7, taking into account the amplitude and phase errors $e_{a}[n]$ and $e_{p}[n]$ calculated at each time step $n$ by Eqs. 5 and 4:

$$
e_{a}[n]=\left|\frac{P_{u}\left(f_{0}\right)}{P_{d}\left(f_{0}\right)}\right|_{m}[n]-\left|\frac{P_{u}\left(f_{0}\right)}{P_{d}\left(f_{0}\right)}\right|_{c}[n]
$$




$$
e_{p}[n]=\angle\left(\frac{P_{u}\left(f_{0}\right)}{P_{d}\left(f_{0}\right)}\right)_{m}[n]-\angle\left(\frac{P_{u}\left(f_{0}\right)}{P_{d}\left(f_{0}\right)}\right)_{c}[n]
$$

where $m$ and $c$ subscripts indicate the "measured" and "command" quantities respectively. Alternatively, we want to make possible the control of the absolute amplitude of $P_{u}$ (independently from $P_{d}$ ). In this case, the new error function $e_{a}^{\prime}[n]$ can be written as follows:

$$
e_{a}^{\prime}[n]=\left|P_{u}\left(f_{0}\right)\right|_{m}[n]-\left|P_{u}\left(f_{0}\right)\right|_{c}[n],
$$

Two PID controllers defined by Eqs. 6 and 7 are implemented using two Max/MSP subpatches allowing for independent control of the amplitude and phase of the complex ratio $P_{u} / P_{d}$ at $f_{0}$.

$$
\begin{aligned}
& A[n]=K_{p a} e_{a}[n]+\frac{K_{i a}}{f_{s}} \sum_{k=0}^{n} e_{a}[k]+K_{d a} \frac{e_{a}[n]-e_{a}[n-1]}{T_{s}}, \\
& \phi[n]=K_{p p} e_{p}[n]+\frac{K_{i p}}{f_{s}} \sum_{k=0}^{n} e_{p}[k]+K_{d p} \frac{e_{p}[n]-e_{p}[n-1]}{T_{s}},
\end{aligned}
$$

where $f_{s}$ is the sampling frequency. The PID coefficients $K_{p n}, K_{i n}$ and $K_{d n}$ (where $n=a$ for the amplitude and $n=p$ for the phase) are tuned empirically to allow for a fast response of the system, while maintaining stability and convergence of PID outputs. In case of a control on $\left|P_{u}\left(f_{0}\right)\right|$ instead of the pressure amplitude ratio, $e_{a}$ is simply replaced by $e_{a}^{\prime}$ in Eq.6.

\subsection{Experimental setup}

The experimental set-up includes a downstream pressure transducer (Endevco $\left.{ }^{\odot} 8507 \mathrm{C}-2\right)$ mounted in the mouthpiece cup, an upstream pressure transducer $\left(\right.$ Endevco $^{\odot}$ 8507C-5) mounted in the mouth cavity just upstream from the lips, and a compression driver $\left(\right.$ AURA $^{\oplus} 3$ inch high-output miniature woofer) mounted on a glass fiber chamber. This allows the output diameter of the driver to be reduced and connected to the mouth cavity. Audio input and output signals are managed by the same audio interface $\left(\mathrm{MOTU}^{\odot}\right)$ running at $44.1 \mathrm{kHz}$. Prior to experiments a relative calibration of the two pressure transducers is performed. The two sensors are mounted on a aluminium tap closing a $30 \mathrm{~cm}$ long cylinder of diameter approximately $30 \mathrm{~mm}$. At the other end of the cylinder, a compressor driver is fixed and a sweep generated between $10 \mathrm{~Hz}$ and $1 \mathrm{kHz}$. The amplitude ratio of the upstream to downstream transducer signals is found around 3.65 across 
the frequency range investigated. This value is then taken into account in the real-time control procedure. Commands of the artificial player are communicated through the $\mathrm{dSpace}{ }^{\odot}$ module working at $1 \mathrm{kHz}$. During acquisition, the quasi-static mouth pressure, as well as the force applied on the lips are recorded using the $\mathrm{dSpace}^{\odot}$. Furthermore, because no accurate gain adjustment is permitted by the gain knobs of the I/O audio interface, a Max/MSP subpatch was used before acquisition in order to calculate two correction coefficients. These coefficients, assigned to the corresponding channels, allow the resulting gains applied to the two input signals to be strictly identical.

\subsection{Experimental protocol}

In the proposed protocol, the amplitude of the upstream acoustic pressure is controlled so that it remains constant, regardless of variations in the downstream acoustic pressure produced. This condition aims at maintaining constant the acoustic energy generated on the upstream side of the lips at $f_{0}$. Experiments are then conducted according to the following sequence of events:

1. Find a proper control parameter setting (input volume flow, lip tension) to obtain a steady and clear tone without active control.

2. Preset active control parameters to the observed phase difference between $P_{u}\left(f_{0}\right)$ and $P_{d}\left(f_{0}\right)$ without active control (which results from the weak acoustical coupling with the mouth cavity).

3. Set a command value for $\left|P_{u}\left(f_{0}\right)\right|$ (constant upstream acoustic energy condition) or for $\left|\frac{P_{u}\left(f_{0}\right)}{P_{d}\left(f_{0}\right)}\right|$ (constant $Z_{u}$ to $Z_{d}$ amplitude ratio condition).

4. Turn the active control on.

5. While maintaining a constant amplitude condition, sweep linearly the phase difference $\angle P_{u}\left(f_{0}\right)-$ $\angle P_{d}\left(f_{0}\right)$ over a range of $240^{\circ}$ in $60 \mathrm{~s}$, which is equivalent to sweeping the phase difference at a rate of $4 \%$ s.

In these experimental conditions, satisfactory performances of the PID controllers are empirically obtained by tuning the coefficients to the following values: $K_{p a}=K_{p p}=0.5, K_{i a}=K_{i p}=0.06$, and $K_{d a}=K_{d p}=0$. Because vocal-tract influence is expected in the higher register of the instrument as shown by measurements on human subjects [4], we try to focus on the highest tones that can be produced by the artificial player without active upstream feedback. 


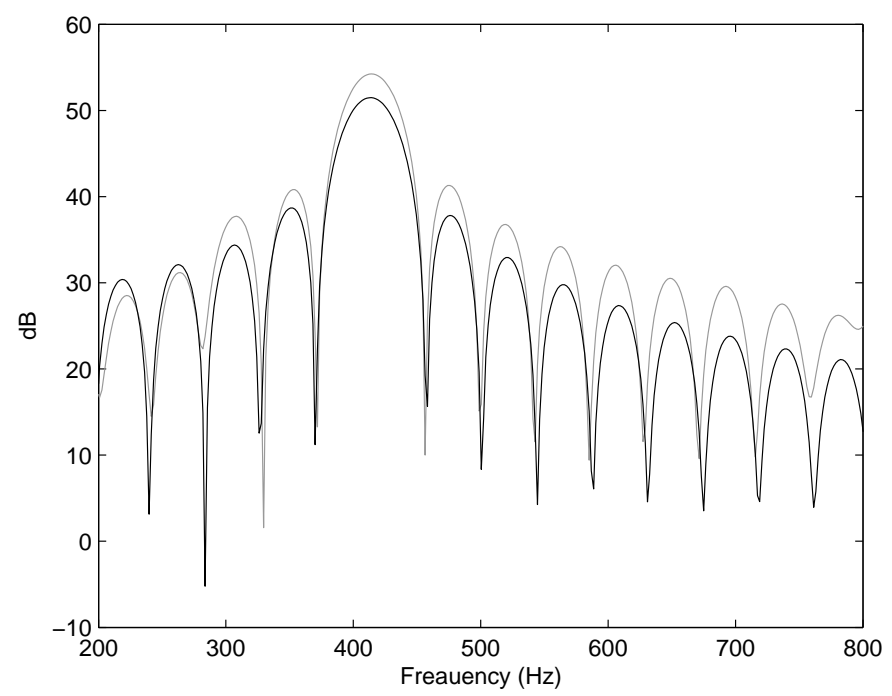

Figure 3: Amplitude spectra around the fundamental frequency of the downstream (gray) and upstream (black) pressures normalized to the maximum of downstream pressure.

Note that the performance of the proposed method is strongly dependent on the accuracy of the $f_{0}$ detection. To verify that the injected upstream signal has the same fundamental frequency as the downstream pressure, the amplitude spectrum of the downstream and upstream pressures monitored during an experiment are calculated over a 1024-sample window taken at a random time step and after zero padding. These spectra, presented in Fig. 3 around the fundamental frequency, show two well-aligned peaks that demonstrate the accuracy of the $f_{0}$ estimation. A peak frequency estimation using parabolic interpolation gives values of $414.2 \mathrm{~Hz}$ and $413.5 \mathrm{~Hz}$ for the fundamental peaks of $P_{d}$ and $P_{u}$, respectively.. This represents $0.18 \%$ error on the estimation of $f_{0}$.

\subsection{Results from experiments}

Data from a 60-second recording with constant upstream energy condition $\left(\left|P_{u}\right|\right.$ is a constant) are presented in Fig. 4. The sounding frequency is close to $415 \mathrm{~Hz}(\mathrm{Ab} 4)$ corresponding to the seventh resonance of the trombone resonator. The input impedance of the trombone used for experiments (measured using the BIAS $^{\odot}$ measurement system [26]) is represented in Fig. 5 along with the location of the playing frequency at time $\mathrm{t}=0 \mathrm{~s}$ and $\mathrm{t}=38 \mathrm{~s}$. In the following, the instantaneous fundamental frequency, phase difference and amplitudes at $f_{0}$ are computed from the spectral analysis of the recorded signals using a moving 1024-point windowing of the signal (512 sample overlap), an FFT analysis applied to the windowed samples after zero 

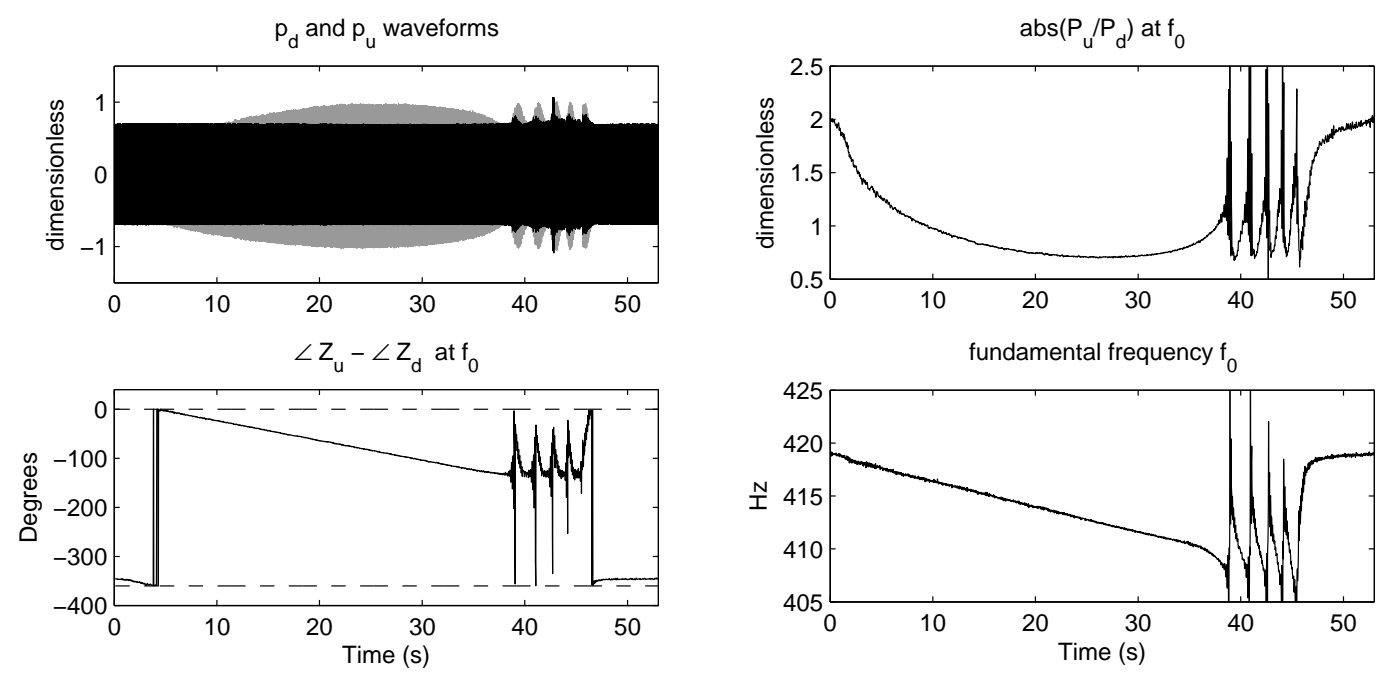

Figure 4: Sustained A4b with linearly varying phase difference between $P_{u}$ and $P_{d}$ at $f_{0}$ and amplitude of $P_{u}$ maintained constant. Left column from top to bottom: $P_{d}$ (gray), $P_{u}$ (black) waveforms and phase difference between $Z_{u}$ and $Z_{d}$ at $f_{0}$. Right column from top to bottom: $P_{u}$ to $P_{d}$ amplitude ratio at $f_{0}$; instantaneous fundamental frequency $f_{0}$.

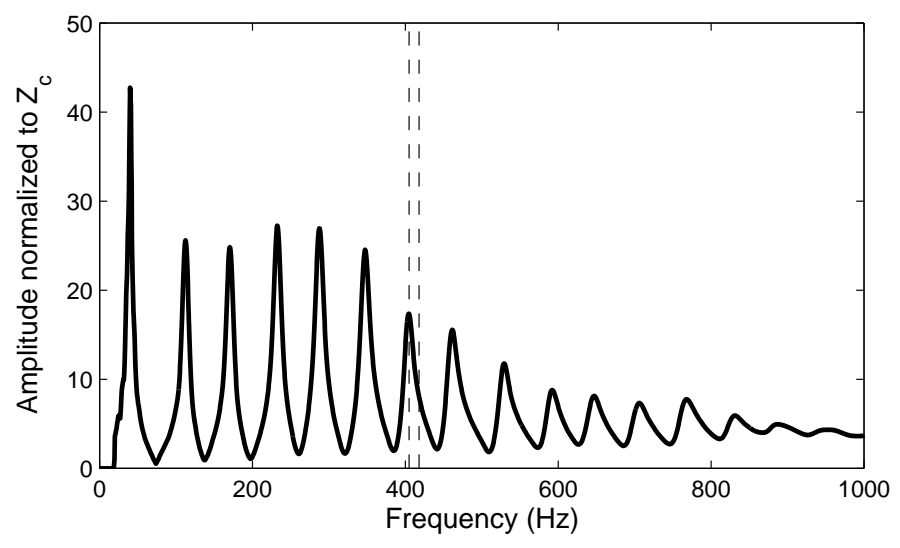

Figure 5: Amplitude of the input impedance of the trombone used for the experiment and normalized to its characteristic impedance $Z_{c}$. The vertical dotted line indicates the playing frequency at time $\mathrm{t}=0 \mathrm{~s}$ $\left(f_{0}=418 \mathrm{~Hz}\right)$ and $\mathrm{t}=38 \mathrm{~s}\left(f_{0}=405 \mathrm{~Hz}\right)$.

padding (by 24000 samples), and parabolic peak interpolation.

From Fig. 4, we first notice that the linear sweep of the phase difference $\angle Z_{u}-\angle Z_{d}$ and the constant upstream acoustic energy are well achieved over the first 38 seconds. The system maintains stable oscillations for 38 seconds before entering into a more unstable regime, certainly due to the target (destructive) phase tuning imposed by the controller at this time, and not supported by the lips. At time 46 seconds, active control is stopped and the lip oscillations return to their initial sustained condition. 
Regarding the stable sounding section (from 0 to $38 \mathrm{~s}$ ), we observe that, despite a constant acoustic energy on the upstream side, the linear phase shift induces significant $f_{0}$ variations from $418 \mathrm{~Hz}$ to $408 \mathrm{~Hz}$.

These results can be further analysed with regards to variations of the downstream input impedance $Z_{d}$, upstream input impedance $Z_{u}$ and total series impedance $Z$ at the fundamental frequency of the sound. From Eq. 1, the complex value of $Z_{u}$ can be derived at frequencies where acoustic energy is observed from the measurement of $Z_{d}$.

The temporal evolution of the phase and amplitude of $Z_{d}, Z_{u}$ and the total impedance loading the lips $Z=Z_{d}+Z_{u}$ at $f_{0}$ are displayed in Fig. 6. Variations of the playing frequency observed in Fig. 4 (decrease in $f_{0}$ with time) results in an increase in $Z_{d}$ amplitude with time. The constant upstream acoustic energy condition results in a ' $U$ ' shape variation of $\left|Z_{u}\right|$ amplitude at $f_{0}$; the magnitude of $Z_{u}$ is higher than $Z_{d}$ for the first $9 \mathrm{~s}$, therefore providing the main support to lip oscillations. Past this point, $\left|Z_{u}\right|$ remains below $\left|Z_{d}\right|$ before growing again until the unstable regime at $\mathrm{t}=38 \mathrm{~s}$. The amplitude of the resulting total impedance $Z$ decreases with time. The linear phase sweep of $\angle P_{u}-\angle P_{d}$ results in a linear increase of the distance between $\angle Z_{u}$ and $\angle Z_{d}$. Both phases of $Z_{d}$ and $Z_{u}$ remain negative during the experiment, indicating a compliant coupling with the downstream and upstream systems. These characteristics of the upstream and downstream impedances are favorable to an outward striking mechanism of the lip-valve system [27].

At time $\mathrm{t}=0 \mathrm{~s}, \angle Z_{d}$ and $\angle Z_{u}$ are very close and at time $\mathrm{t}=4 \mathrm{~s}, Z_{d}$ and $Z_{u}$ are in phase. This constructive phase tuning in the beginning of the experiment contributes to the amplitude of the total impedance $Z$. The slight increase of $\angle Z_{d}$ and significant decrease in $\angle Z_{u}$ cause a marked decrease of $\angle Z$ with time. We notice that at time $\mathrm{t}=20 \mathrm{~s}, \angle Z=-90^{\circ}$. The lips enter an unstable regime of oscillation when $\angle Z_{u}$ reaches $-180^{\circ}$. The active control forces $\angle Z_{u}$ towards a phase reversal to $+180^{\circ}$ that the lip oscillator does not seem to support. This instability may be caused by the transition from a capacitive to an inductive upstream coupling, the latter being potentially unfavorable to the mechanism of lip oscillation. This hypothesis may be drawn in conjunction with the large value of the phase difference between $Z_{d}$ and $Z_{u}$ reached when the unstable regime is initiated.

Finally and most importantly, we observe that the downstream pressure at $f_{0}$ follows a bell-like curve showing a maximum value indicating a potential optimum tuning of the system. Furthermore, and despite the variations of playing frequency inducing changes in $\left|Z_{d}\right|$ at $f_{0}$, this maximum does not match with a $\left|Z_{d}\right|$ peak value. This observation hence suggests that this optimal tuning point results not only from an increase in downstream acoustical feedback, but also from a beneficial phase tuning of $Z_{u}$ relative to $Z_{d}$ at 

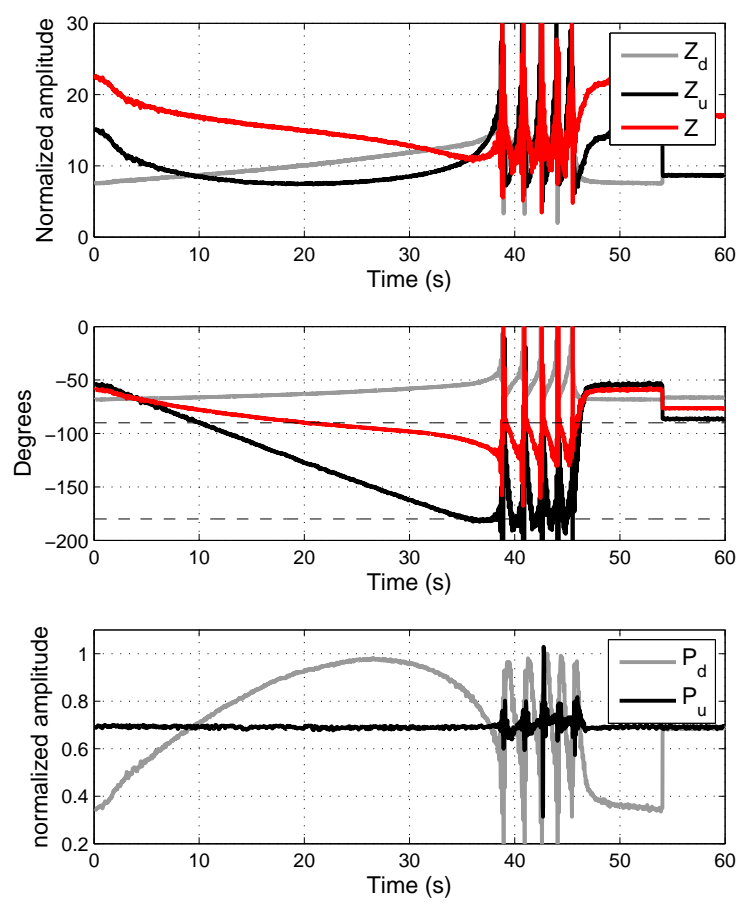

Figure 6: Temporal evolution of $Z_{d}, Z_{u}$ and $Z$ at the playing frequency $f_{0}$ in the constant upstream energy condition. Amplitude (top) and phase (middle) of $Z_{d}$ measured experimentally (light gray), as well as $Z_{u}$ (black) and $Z$ (gray - red in color version). The two horizontal dashed lines indicate -90 and $-180^{\circ}$ phase angles. Bottom: $P_{d}$ (gray) and $P_{u}$ (black) amplitudes at $f_{0}$ normalized to the maximum of $P_{d}$. 
$f_{0}$. It further suggests that an optimal upstream phase tuning allows the playing frequency to maximize the lip motion and hence generate a larger acoustic volume flow into the instrument. Because of the frequency shift observed, this might arise from the displacement of the playing frequency near a mechanical resonance of the lips.

In order to investigate the influence of upstream energy amplitude on the linear decrease of $f_{0}$, we repeated our experiment with different levels of upstream acoustic energy. The upstream pressures are normalized to the highest upstream pressure used. Using this convention, results presented in Fig. 4 and 6 refer to an $85.6 \%$ upstream pressure. Results obtained for the three different conditions $(59.0 \%, 85.6 \%$ and 100\%) are summarized in Table 1. Increasing the amplitude of the upstream signal has a significant effect on variations of the playing frequency. Meanwhile, the maximum of downstream energy occurs around $414 \mathrm{~Hz}$ in the three conditions for a phase shift value between $P_{u}$ and $P_{d}$ close to $90^{\circ}$. While the air supply feeding the mouth cavity is set to a constant input flow, the quasi-static pressure $p_{0}$ in the mouth was found to reach a minimum when the amplitude of $P_{d}\left(f_{0}\right)$ is maximum. However, these variations remain small with $\Delta p_{0}=3 \%$ of the maximum value of $p_{0}$ for the highest upstream level condition.

The maximum and minimum values of the upstream to downstream pressure ratios, inversely related to the amplitude ratio of the upstream to downstream input impedances, are reported in Table 1. Since $\left|P_{u}\right|$ is maintained constant, the value of $\min \left(\left|\frac{P_{u}}{P_{d}}\right|\right)$ occurs when $\left|P_{d}\right|$ is maximum. This observation highlights the fact that a high upstream impedance amplitude does not necessarily contributes positively to the regeneration process. For instance, the maximum efficiency of the system occurs when the vocal-tract only contributes half of the trombone acoustical feedback in the 59.0\% condition. Finally, a fourth condition is simulated where the amplitude ratio $\left|\frac{P_{u}}{P_{d}}\right|$ is maintained constant at $f_{0}$ (see Table 1). This condition leads to relatively similar results with maximum downstream energy produced at $414.0 \mathrm{~Hz}$ and variations of $p_{0}$ over $2.2 \%$ of the maximum value.

In order to further understand the nature of the optimal tuning point identified in the previous section, we propose to work under even more controlled conditions through numerical simulations. We hence investigate the influence of upstream coupling on two physical models of the lips: a traditional one-dimensional model which can be parametrized to oscillate either according to an outward or inward striking mechanism, and a two-dimensional physical model proposed by Adachi and Sato [22] and represented in Fig. 10. 


\begin{tabular}{ccccccc}
\hline \hline Condition & $\Delta f_{0}$ & $f\left(P_{d_{\max }}\right)$ & $\phi\left(P_{d_{\max }}\right)$ & $\max \left(\left|\frac{P_{u}}{P_{d}}\right|\right)$ & $\min \left(\left|\frac{P_{u}}{P_{d}}\right|\right)$ & $\Delta p_{0}$ \\
\hline$\left|P_{u}\right|=59.0 \%$ & $6.5 H z$ & $414.5 \mathrm{~Hz}$ & $-88^{\circ}$ & 1.0 & 0.54 & $1.6 \%$ \\
$\left|P_{u}\right|=85.6 \%$ & $10 H z$ & $414.2 \mathrm{~Hz}$ & $-90^{\circ}$ & 2.0 & 0.70 & $2.5 \%$ \\
$\left|P_{u}\right|=100 \%$ & $14 H z$ & $414.1 \mathrm{~Hz}$ & $-91^{\circ}$ & 3.0 & 0.78 & $3.0 \%$ \\
$\left|\frac{P_{u}}{P_{d}}\right|=1$ & $8.5 H z$ & $414.0 H z$ & $-92^{\circ}$ & 1.1 & 0.96 & $2.2 \%$ \\
\hline \hline
\end{tabular}

Table 1: $f_{0}$ variations, frequency and phase $\phi$ at which the downstream pressure is maximum, maximum and minimum values of $P_{u}$ to $P_{d}$ amplitude ratio at $f_{0}$, and quasi-static mouth pressure variations (in $\%$ of the maximum value of $p_{0}$ recorded during the stable part of the tone), extracted in three conditions of constant upstream energy (normalized to the condition of maximum upstream energy) and one condition of constant upstream to downstream energy ratio. For all conditions, $\phi$ was swept linearly over a range of $145^{\circ}$.

\section{One-dimensional outward striking model}

\subsection{Description of the model}

The mechanical model chosen for the lips is inspired from previous studies [27, 32, 28]. The two lips are assumed to be identical and placed symmetrically on the mouthpiece. Each lip is allowed to move along a vertical axis and its vertical position relative to the equilibrium position $y_{0}$ is denoted $y$. To simplify the problem, the length of the lip channel is considered close to zero and therefore no interactions are considered between the jet and the channel face. The dynamics of each lip can be represented by the simple secondorder oscillator equation:

$$
\frac{d^{2} y}{d t^{2}}+\frac{\omega_{l i p}}{Q_{l i p}} \frac{d y}{d t}+\omega_{l i p}^{2}\left(y-y_{0}\right)=\frac{F}{m_{l i p}},
$$

where $\omega_{l i p}$ is the lip angular frequency $\left(\omega_{l i p}=2 \pi f_{l i p}\right), Q_{l i p}$ the quality factor, $m_{l i p}$ the mass of one lip and $y_{0}$ is the vertical equilibrium position of the lip. $F$ is the vertical component of the force acting on the lip. $F$ is proportional to the pressure difference between mouth and mouthpiece i.e. $F / m_{\text {lip }}=\left(p_{0}+p_{u}-p_{d}\right) / \mu$, where $\mu$ is the effective mass of the lips, $m_{\text {lip }}$, divided by the effective area of the internal face of the lips; this force therefore supports an outward striking reed behaviour.

The lip channel is assumed to have constant width $b$ so that the time-varying lip opening area $s_{l i p}(t)$ is given by the expression $s_{l i p}(t)=2 b\left(y_{0}+y(t)\right)$. The volume-flow $u(t)$ through this channel is assumed to be quasi-stationary, frictionless and incompressible. Furthermore, the area of the mouthpiece entry $s_{c u p}$ is supposed much larger than the lip opening area over a full cycle $\left(s_{\text {lip }}<<s_{\text {cup }}\right)$, so no pressure recovery 
occurs at the mouthpiece cup. Under these assumptions, the volume flow $u(t)$ can be expressed as a function of the pressure difference across the lips $p_{0}+p_{u}(t)-p_{d}(t)$ using a Bernoulli flow model:

$$
u(t)=\alpha \cdot \sqrt{\frac{2\left|p_{0}(t)+p_{u}(t)-p_{d}(t)\right|}{\rho}} \cdot 2 b\left(y_{0}+y(t)\right),
$$

where $\alpha=\operatorname{sgn}\left(p_{0}(t)+p_{u}(t)-p_{d}(t)\right), \rho$ is the average air density and $\operatorname{sgn}(x)=+1$ if $x$ is positive or null and -1 if $x$ is negative.

\subsection{Downstream and upstream coupling}

While the downstream coupling is taken into account through the measured trombone input impedance $Z_{d}$ or its inverse Fourier transform $z_{d}\left(p_{d}=z_{d} * u\right)$, the upstream coupling is implemented by disturbing the system through the injection of a sinusoidal upstream acoustic pressure $p_{u}(t)$ of the same instantaneous fundamental frequency as $p_{d}(t)$. Similarly to the in-vitro experiment reported in Section 2, the amplitude $p_{u}(t)$ is maintained constant while the instantaneous phase of $p_{u}(t)$ relative to the phase of $p_{d}(t)$ at $f_{0}$ is varied along the tone duration. This results in a linear variation of the phase difference $\angle Z_{u}\left(f_{0}\right)-\angle Z_{d}\left(f_{0}\right)$.

The numerical implementation of this dynamic upstream tuning involves a looped sequence which includes the following steps:

1. After sample $p_{d}(n)$ has been calculated, a Butterworth second-order bandpass filter (with lower and higher cutoff frequencies $f_{l i p}-100 \mathrm{~Hz}$ and $f_{l i p}+100 \mathrm{~Hz}$ respectively) is applied to the vector $-p_{d}$. This is performed through a zero-phase forward and reverse filtering process to avoid phase shifts induced by the filter using the matlab function filtfilt.m. Note that the resonance bandwidth of the filter is chosen much smaller than the spacing between harmonics of the expected fundamental. The resulting vector, named $p_{s}$, is therefore a sinusoidal waveform of amplitude given by the resonant filter coefficients and out of phase with $p_{d}\left(f_{0}\right)$ by $180^{\circ}$ at $f_{0}$.

2. $p_{s}$ is normalized to its maximum value over its two last periods and scaled by a constant factor $C$. This step enables the amplitude of acoustic upstream energy to be maintained constant, independently from the amplitude of the downstream pressure produced.

3. $p_{u}(n)$ is set from the normalized and scaled value of $p_{s}$, with a time-shift given by a phase-shift parameter $\Phi(n) ; p_{u}(n)=p_{s}[n-\Phi(n)]$. The phase shift parameter $\Phi(n)$ (implemented as a delay in 
samples) is an input parameter which thus controls the phase difference between $P_{d}$ and $P_{u}$.

4. $p_{u}(n)$ is applied as in Eqs. 8 and 9.

5. The procedure is iterated by one time step.

\subsection{Results from simulations}

Simulations are performed using the following parameter values adapted from previous simulations [28]: $f_{\text {lip }}=310 \mathrm{~Hz}, Q_{\text {lip }}=6, \mu=25 \mathrm{~kg} / \mathrm{m}^{2}, b=10 \mathrm{~mm}, y_{0}=0 \mathrm{~mm}, s_{\text {cup }}=4.8 \mathrm{~cm}^{2}, \rho=1.1769 \mathrm{~kg} / \mathrm{m}^{3}$, $c=347.23 \mathrm{~m} / \mathrm{s}$. It is worth mentioning that in the trombone configuration chosen, the fifth and the sixth resonances of the downstream input impedance are around $290 \mathrm{~Hz}$ and $350 \mathrm{~Hz}$ respectively. It is then clear that the chosen lip resonance frequency $f_{l i p}$ is between two acoustic resonances, rather than very close to one, hence allowing oscillations to occur potentially near one or the other downstream resonance.

Time-domain simulations are performed by discretization of the differential equations using the forward Euler method and applying the trapezoidal approximation for the integration in the downstream coupling equation as performed in [32]. The sampling frequency is set to $48 \mathrm{kHz}$.

The quasi-static pressure $p_{0}$ is specified using a quarter-period sinusoidal onset of $10 \mathrm{~ms}$, sustain at $15 \mathrm{kPa}$, and a quarter-period sinusoidal decay of $10 \mathrm{~ms}$. The phase shift vector $\Phi$ contains values varying linearly from 110 to $240^{\circ}$; the value contained in $\Phi$ at index $n$ indicates the number of shifted samples applied to $p_{s}$ (in order to obtain $p_{u}$ value at index $n$ ). It therefore refers to the instantaneous phase shift between $p_{u}$ and $p_{d}$ waveforms at $f_{0}$. The boundary values in $\Phi$ are chosen empirically so that the phase shift between $p_{u}$ and $p_{d}$ covers the maximal range allowing the system to maintain oscillations. Scaling parameter $C$ is set so that the $p_{u}$ amplitude is of the same order of magnitude as $p_{d}$, hence producing significant effect on the system. Such orders of magnitude of $P_{u}\left(f_{0}\right) / P_{d}\left(f_{0}\right)$ amplitude ratio are observed in trombone players in the higher register [4]. A quarter-period sinusoidal onset envelope is applied to the scaling factor so that the $p_{u}$ amplitude grows smoothly from zero to $C$ when injected into the system.

Simulations are initiated with no upstream feedback $\left(p_{u}=0\right)$. Once the permanent regime of oscillation is reached (time $t_{1}$ determined empirically), an acoustic upstream pressure component is added to $p_{0}$ according to the procedure described in the previous section.

The waveforms of $p_{d}$ and $p_{u}$ calculated from a two-second simulation are presented in Fig. 7. The upstream feedback is introduced around $0.6 \mathrm{~s}$ and induces significant variations in $p_{d}$ amplitude 

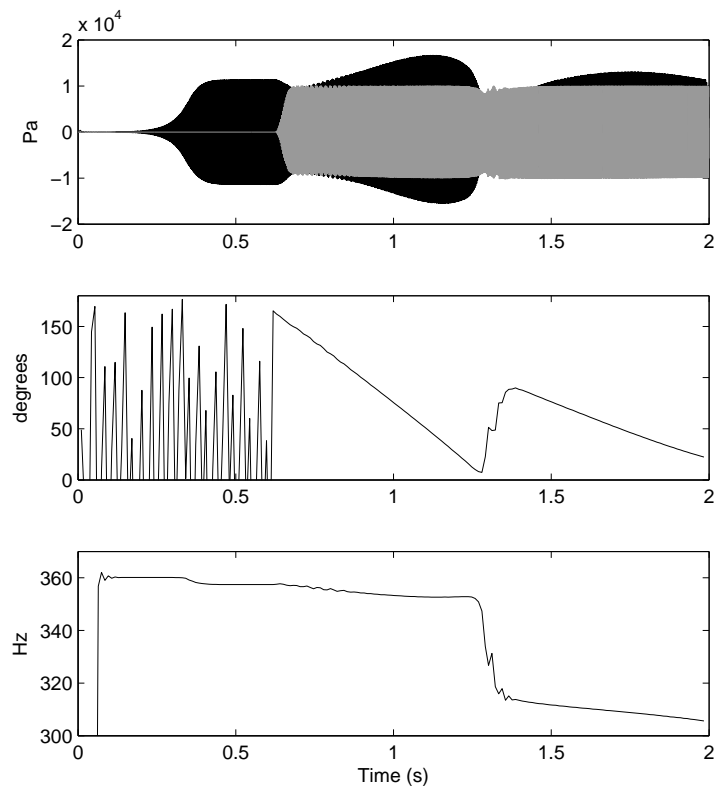

Figure 7: Results from a 96000 sample simulation at $48 \mathrm{kHz}$ for an outward striking lip model. From top to bottom: waveforms of $p_{d}$ (black) and $p_{u}$ (gray); phase difference between $P_{u}$ and $P_{d}$ at the fundamental frequency; fundamental frequency $f_{0}$.

and $f_{0}$ as the phase difference $\angle P_{u}-\angle P_{d}$ varies linearly in time at $f_{0}$. At time $1.3 \mathrm{~s}$, a register transition to a lower mode is observed. It results in a smaller slope of the phase shift due to a smaller value of $f_{0}$. As for the higher mode, $f_{0}$ decreases slowly while $\angle P_{u}-\angle P_{d}$ is decreased until the end of the tone. Comparing these results with the one obtained using active control on the artificial player system (Fig. 4), we observe that the two sets of data share common features: across the two portions of stable oscillations in Fig. 7, the downstream waveform goes through an amplitude maximum for a given phase tuning. Furthermore, the linear phase shift results in a linear decay of the playing frequency in both artificial player and numerical model. For the model, this linear variation particularly occurs for the lower tone, which is also close to the lip natural frequency $f_{\text {lip }}$.

\subsection{Lip mobility calculation}

Considering only the alternating components of the different variables, the analytical expression of the lip mobility can be deduced from Eq. 8 as the transfer function of a simple one-dimension second-order oscillator: 

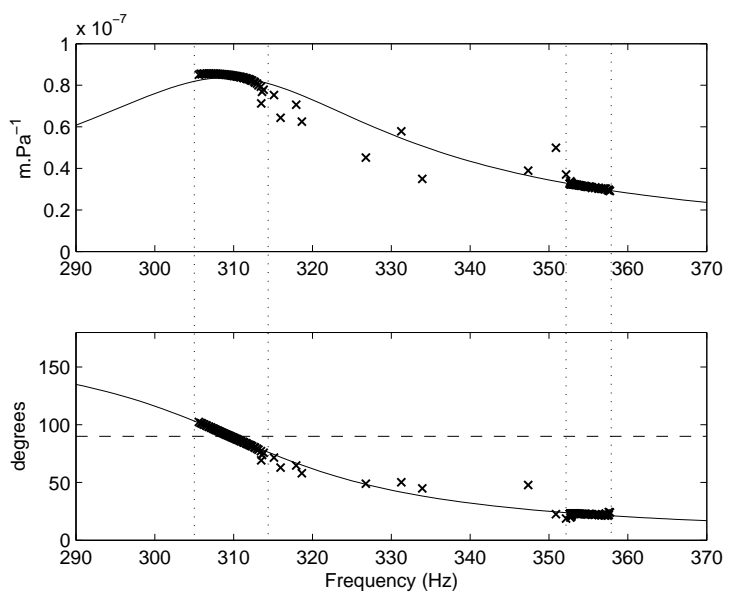

Figure 8: Calculated and simulated lip mobility for an outward striking model. Amplitude (top) and phase (bottom) of $G$ : theoretical value (solid line) and calculated from numerical simulations (cross). The horizontal dashed line indicates $90^{\circ}$ phase angle. The vertical dotted lines indicate the two regions of stable oscillations corresponding to the two produced tones.

$$
G(\omega)=\frac{Y(\omega)}{\Delta P(\omega)}
$$

where $\Delta P$ is the Fourier representation of the pressure difference across the lips $p_{d}-p_{u}$.

The magnitude and phase of $G$ are represented by solid lines in Fig. 8. As predicted by the mechanical model, the outward striking character of the lip-valve system results in a $+90^{\circ}$ phase shift between $Y$ and $\Delta P$ at the resonance.

In the same figure, the amplitude and phase of the lip mobility at $f_{0}$ calculated from simulated waveforms of $p_{d}, p_{u}$ and $y$ are represented in crosses (note that the thick black lines result from a large number of superimposed crosses). In agreement with the model, and over the frequency range where acoustic energy is observed, the amplitude and phase of $G$ estimated from simulations overlap with their theoretical values. In this particular situation, variations of $G$ at $f_{0}$ caused by the upstream feedback allow oscillations to occur over a significant frequency range. For the second regime $(t>1.3 \mathrm{~s})$, the swept range includes the lip natural frequency identified by $\angle G\left(f_{0}\right)=90^{\circ}$. At the lip resonance frequency, the lip motion is maximized, hence increasing the volume flow generated in the instrument. This first observation thus provides an important element supporting the hypothesis of an optimal phase tuning of the vocal tract at $f_{0}$ with respect to the motion of the lips. For the first regime, the maximization of the downstream energy seems mainly caused by an increase of the magnitude of $Z_{d}$ at $f_{0}$. 

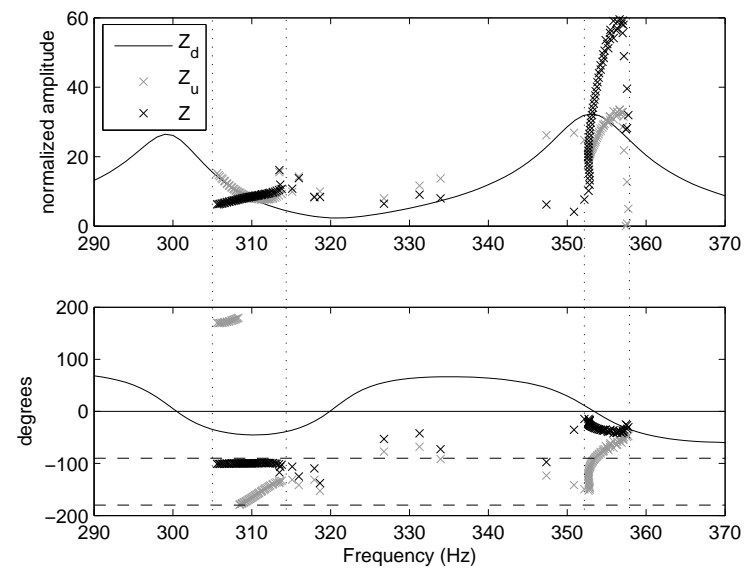

Figure 9: $Z_{d}, Z_{u}$ and $Z$ during simulations for an outward striking model. Amplitude (top) and phase (bottom) of $Z_{d}$ obtained from measurements (solid line), as well as $Z_{u}$ (gray cross) and $Z$ (black cross) at frequencies where acoustic energy is observed. The two horizontal dashed lines indicate $-90^{\circ}$ and $-180^{\circ}$ phase angles. The vertical dotted lines indicate the two regions of stable oscillations corresponding to the two produced tones.

\subsection{Downstream and upstream input impedances}

The influence of downstream and upstream acoustical feedback can be further discussed in terms of input impedances $Z_{d}$ and $Z_{u}$. The total impedance "loading" the lip-valve $Z=U / \Delta P$ can be expressed from Eq. 1 as the sum of the downstream and upstream impedances: $Z=Z_{d}+Z_{u}$.

The amplitude and phase of $Z_{d}$, as well as the amplitude and phase of $Z_{u}$ and $Z$ at $f_{0}$ calculated at each time step from the value of $Z_{d}$ and from Eq. 1 are represented in Fig. 9. In this frequency-domain representation, the temporal information is lost, but the effect of the upstream feedback on the impedance quantities at $f_{0}$ is made visible over the frequency range covered. More specifically, this figure illustrates the influence of the disturbing upstream signal on both amplitude and phase of the upstream, downstream and total impedances charging the lips at $f_{0}$.

It can be seen that in this particular case, the upstream coupling tends to lower the value of $\angle Z$. As $\angle Z$ becomes closer to zero and as $|Z|$ is significantly decreased, oscillations become unstable. When $f_{0}$ reaches $353 \mathrm{~Hz}$, a change of regime then occurs from the $7^{\text {th }}$ to $6^{\text {th }}$ acoustic mode.

These observations can be further discussed in light of the linear theory of oscillation. The phase condition of regeneration under which sustained oscillations are maintained is given by Eq. 11 [29]: 


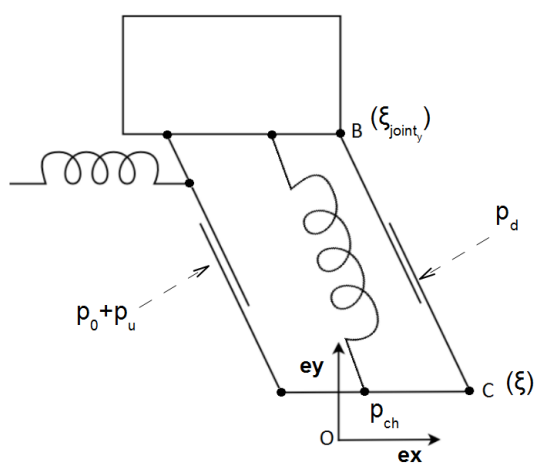

Figure 10: Two-dimensional model of the lips proposed by Adachi and Sato [22].

$$
\angle Z\left(f_{0}\right)+\angle G\left(f_{0}\right)=0
$$

where $G$ is defined by Eq. 10. This phase condition relies on the linearisation of the flow equation around the quasi-static pressure $p_{0}$ (Eq. 9) and therefore supposes that $s_{l i p}(t)$ and $u(t)$ oscillate in phase at the playing frequency.

Assuming that Eq. 11 governs the system when oscillations are established, we observe that injection of upstream acoustic energy allowing $\angle Z$ to reach values below $-90^{\circ}$ enables $\angle G$ to be displaced above $90^{\circ}$ at $f_{0}$. It thus enables oscillations to occur both above and below the lip natural frequency $f_{\text {lip }}$. In the $353-358 \mathrm{~Hz}$ region, the lowered value of $\angle Z$ enables oscillations to occur at a lower frequency and hence closer to a maximum in $\left|Z_{d}\right|$. In the $305-315 \mathrm{~Hz}$ region, the upstream coupling enables $\angle Z$ to be displaced around $-100^{\circ}$ and oscillations to occur at the lip natural frequency $f_{\text {lip }}$ for which $\angle G=+90^{\circ}$. In spite of the non-linearity induced by the downstream and upstream feedback, in the flow equation, Eq. 11 qualitatively explains the displacement of the playing frequency close to the lip resonance caused by appropriate upstream phase tuning conditions. Consequently, the combined effect of an increase in the amplitude of $G$ and $Z_{d}$ at $f_{0}$ induces a maximum of acoustic pressure generated on the downstream side of the lips. 


\section{Two-dimensional lip model}

\subsection{Mechanical and flow equation}

This representation allows motion of the lips in both longitudinal and transverse direction, therefore making possible both outward and upward movements as observed in real lips [30, 31]. Contrary to simple outward and inward striking models [27, 32], this representation presents an important non-linear character arising from the time-varying internal and external lip surface areas. This characteristic is responsible for the ability of the model to operate according to both inward and outward striking regimes. The mechanical equation is given by Eq. 12:

$$
\frac{1}{2} m_{l i p} \frac{d^{2} \xi}{d t^{2}}+\frac{1}{2} \frac{\sqrt{m_{l i p} k}}{Q_{l i p}} \frac{d \xi}{d t}=\boldsymbol{F}_{r}+\boldsymbol{F}_{\Delta p}+\boldsymbol{F}_{B}
$$

where $k$ is the stiffness factor of the lips, $\xi$ is a two dimensional vector whose coordinates are given by the position of point $C$ in the direct orthonormal system $(0 ; \boldsymbol{e x} ; \boldsymbol{e y})$, and $\xi_{\text {equil }}$ is the two-dimensional equilibrium vector position (note that $B$ has a zero-value $x$ position). $\boldsymbol{F}_{r}=-\frac{1}{2} k\left(\xi-\xi_{\text {equil }}\right.$ ) is the elastic restoring force pointing to $\xi_{\text {equil }}$ and arising from the combined action of the two springs of the same stiffness (see Fig. 10). $\boldsymbol{F}_{\Delta p}=s\left(p_{0}+p_{u}-p_{d}\right) \mathbf{n}$ is the external force acting on the internal and external lip faces due to the pressure difference across the lip valve, where $\mathbf{n}$ is a unitary vector normal to the internal lip face and oriented toward positive horizontal-axis values, $s$ is the time-varying effective area of the internal and external lip faces, and $\theta$ is the angle between $B C$ and the vertical axis defined by $\boldsymbol{e} \boldsymbol{y}$. Finally $\boldsymbol{F}_{B}=b d p_{c h} \mathbf{e y}$ is the force acting on the lip channel face and generated by the Bernoulli pressure, where $d$ is the thickness of one lip and $p_{c h}$ is the pressure in the lip channel.

Under the similar assumption of total dissipation of the kinetic energy of the jet in the mouthpiece as stated in Section 3, the pressure in the mouthpiece is considered equal to the pressure on the lip channel: $p_{c h}=p_{d}$

Elliot and Bowsher proposed simple relationships between $k, m_{l i p}$ and $f_{l i p}$ derived from quasi-static measurements [29]. More specifically, they suggest a proportional relationship between $k$ and $f_{\text {lip }}$, while

$m_{l i p}$ and $f_{l i p}$ hence become inversely proportional $\left(\omega_{l i p}=\sqrt{\frac{k}{m_{l i p}}}\right)$. These relations were later used by Adachi and Sato in their simulations [32, 22]. We therefore chose to use Adachi and Sato formulas to enable further comparisons with their results: $k=1.5 f_{\text {lip }}$ and $m_{\text {lip }}=1.5 /\left((2 \pi)^{2} f_{\text {lip }}\right)$. 
In this model, a second flow component $u_{l i p}(t)$ induced by the longitudinal motion of the lips and equivalent to the volume swept by the lips per unit of time is considered [22] so that the total volume flow entering the system is $u_{T}(t)=u(t)+u_{l i p}(t)$ and $u_{l i p}(t)$ is given by the following expression:

$$
u_{l i p}(t)=b\left[\xi_{x}(t) \frac{d \xi_{y}}{d t}-\left(\xi_{y}(t)-O B\right) \frac{d \xi_{x}}{d t}\right]
$$

\subsection{Results from simulations}

Numerical simulations are performed using the same conditions of downstream and upstream feedback as presented in Section 3.2 and using the following parameters: $f_{l i p}=470 \mathrm{~Hz}, Q_{l i p}=3, b=10 \mathrm{~mm}$, $d=4 \mathrm{~mm}, \xi_{\text {joint }_{y}}=4 \mathrm{~mm} \xi_{\text {equil }_{x}}=2 \mathrm{~mm}, \xi_{\text {equil }_{y}}=0.3 \mathrm{~mm}, s_{\text {cup }}=4.8 \mathrm{~cm}^{2}, \rho=1.1769 \mathrm{~kg} / \mathrm{m}^{3}$, $c=347.23 \mathrm{~m} / \mathrm{s}$. This leads to $m_{\text {lip }}=80.8 \mathrm{mg}$ and $k=705 \mathrm{~N} / \mathrm{m}$. Finally, the phase shift vector $\Phi$ is set to vary between 110 and 230 samples.

When the lips are in contact $\left(s_{l i p}=0\right)$, Adachi and Sato proposed a supplementary restoring force accounting for the collision between the lips, and triggered by the condition $\xi_{y}<0$. Additionally, the viscous loss due to the collision of the lips was taken into account by updating $Q$ to a value smaller than 1 during the closed phase (i.e. when $s_{l i p}=0$ ) [22]. Likewise, Vergez and Rodet proposed to add an extra stiffness coefficient and damping coefficient during the closed phase [23], likely to be dynamically adjusted during the progressive closure [33]. For simplicity, we do not take into account these adjustments and only set $\xi_{y}=0$ when $s_{l i p}=0$. This simplification is equivalent to a re-initialization of $\xi_{y}$ to zero whenever complete closure of the lip valve occurs.

Time-domain simulation results are presented in Fig. 11. Contrary to previous simulations, the amplitude of $p_{u}(t)$ is set through coefficient $C$ to a smaller value since this lip model appears to be more sensitive to an upstream acoustical perturbation. Despite the small amplitude of $p_{u}(t)$, the linear sweep of the phase difference $\angle Z_{u}-\angle Z_{d}$ at $f_{0}$ results in significant variations of the fundamental frequency with a transition to a lower mode between 1.2 and $1.6 \mathrm{~s}$. Along with variations in $f_{0}$, the acoustic downstream energy varies noticeably during sound production.

By only considering the $\mathrm{AC}$ component of the variables involved and neglecting the influence of quadratic terms, the analytical expression of the lip mobility $G(\omega)=\frac{\xi_{y}^{a c}(\omega)}{\Delta P(\omega)}$ can be calculated [22]. The amplitude and phase of the lip mobility $G$ calculated analytically (solid line) and from simulations (cross) are represented in Fig. 12. 

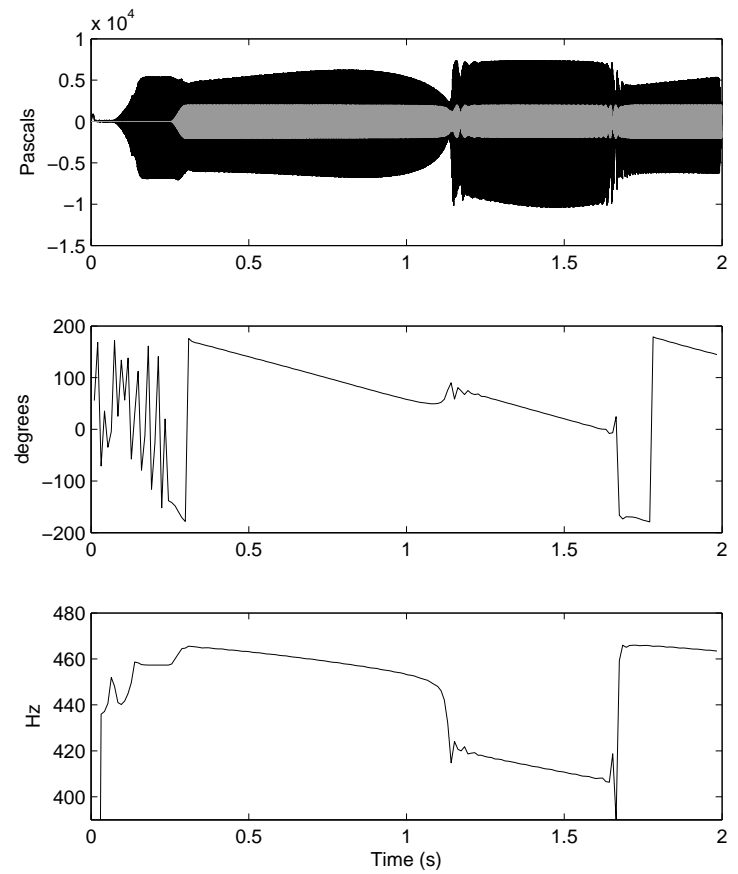

Figure 11: Results from a 96000 sample simulation at $48 \mathrm{kHz}$ for a two-dimensional lip model. From top to bottom: waveforms of $p_{d}$ (black) and $p_{u}$ (gray); phase difference between $P_{u}$ and $P_{d}$ at the fundamental frequency; fundamental frequency $f_{0}$.

Results from simulations weakly agree with the theoretical value of $G$, although $\angle G$ estimated from simulations lies in the region of values defined by the analytical expression of the lip mobility. Regarding the amplitude, values obtained from the time-domain simulation particularly disagree with the theoretical value in the $450-470 \mathrm{~Hz}$ region. These discrepancies may be attributed to the approximations performed in the analytical calculation: static component of the downstream pressure neglected, quadratic term $\xi_{y}^{a c}\left(P_{u}-P_{d}\right)$ omitted, and influence of the upstream acoustic pressure not considered.

However, both simulation and theoretical results agree to predict the change in sign of $\angle G$, indicating a transition from an inward to outward striking regime of oscillation when the playing frequency decreases. The influence of the upstream acoustic pressure taken into account in the calculation of the analytical expression of the lip mobility leads to Eq. 14:

$$
G^{\prime}(\omega)=2 b \frac{\left(\frac{d}{1-P_{u}(\omega) / P_{d}(\omega)}-\xi_{x}^{d c}\right)-2 b\left(\xi_{\text {joint }_{y}}-\xi_{y}^{d c}\right) p_{0}\left[\gamma\left(\omega / \omega_{\text {lip }}\right) / k\right]}{1+\left(2 b p_{0}\left[\gamma\left(\omega / \omega_{\text {lip }}\right) / k\right]\right)^{2}} \frac{\gamma\left(\omega / \omega_{\text {lip }}\right)}{k}
$$

with $\gamma(\omega)=1 /\left(1-\omega^{2}+i\left(\omega / Q_{l i p}\right)\right)$. This equation looks relatively similar to the expression provided in 

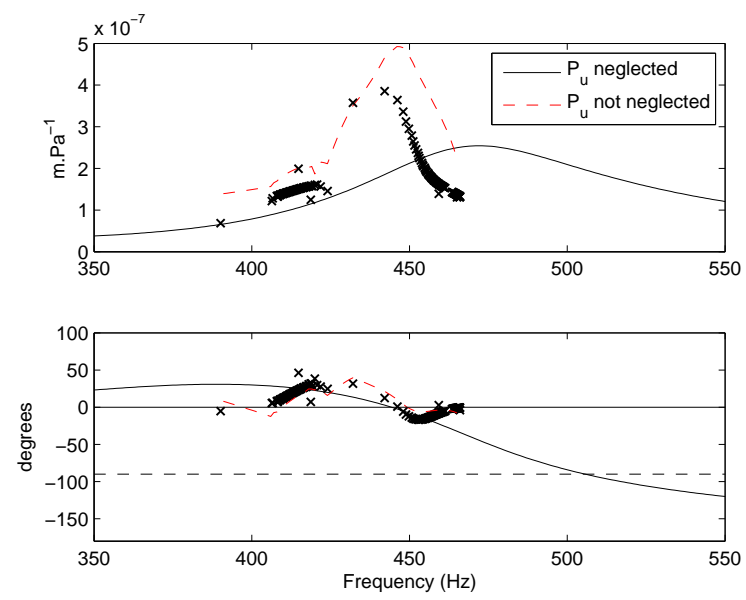

Figure 12: Calculated and simulated lip mobility for a two-dimensional lip model. Amplitude (top) and phase (bottom) of $G$ : theoretical value (solid line), theoretical value corrected by the upstream coupling $\left(G^{\prime}\right)$ (dashed gray - red in color version), and values calculated from numerical simulations (cross). The horizontal dashed line indicates $-90^{\circ}$ phase angle.
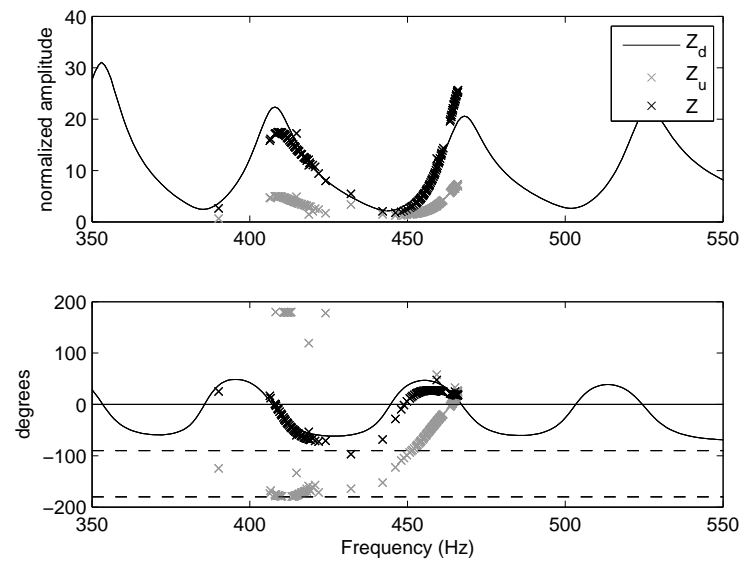

Figure 13: $Z_{d}, Z_{u}$ and $Z$ during simulations for a two-dimensional lip model. Amplitude (top) and phase (bottom) of $Z_{d}$ obtained from measurements (solid line), as well as $Z_{u}$ (gray cross) and $Z$ (black cross) at frequencies where acoustic energy is observed during simulations. The two horizontal dashed lines indicate $90^{\circ}$ and $180^{\circ}$ phase angles. 
[22] but differs in some details: a new term $\frac{1}{1-P_{u} / P_{d}}$ appears in the numerator and reflects the influence of the upstream coupling on the lip mobility; furthermore, the term $\xi_{y}^{d c}$ is taken into account in the numerator while it seems to be neglected in Adachi and Sato's formulation. This expression is thus only computable at frequencies where acoustic energy is produced. The amplitude and phase of this corrected lip mobility $G^{\prime}$ is represented in Fig. 12 (dashed line). Results from simulation are in better agreement with $G^{\prime}$, especially for the phase where simulation results overlap well in the $405-420 \mathrm{~Hz}$ region. Regarding the amplitude, the hypothesis of a maximum in lip mobility amplitude around $445 \mathrm{~Hz}$ suggested by simulation results is somewhat confirmed by the computed magnitude of $G^{\prime}$.

Looking at the amplitude and phase of $Z_{d}, Z_{u}$ and $Z$ in Fig. 13, we observe that the small magnitude of $Z_{u}$ compared to $Z_{d}$ (resulting from the low amplitude of $p_{u}$ ) induces a small effect on the value of $\angle Z$. Consequently, $Z$ is strongly correlated to $Z_{d}$ in amplitude and phase. Despite this small upstream influence, significant variations in $f_{0}$ occur, as observed in Figs. 11 and 12. The linear theory of oscillation provides a reasonable prediction of the relationship between $\angle G$ and $\angle Z$ through Eq. 11; the positive sign of $\angle Z$ over the $450-470 \mathrm{~Hz}$ range correctly agrees with the negative sign of $\angle G$ over the same frequency interval, while the negative sign of $\angle Z$ over the $405-420 \mathrm{~Hz}$ range is in agreement with the positive character of $\angle G$ over the same interval.

Finally, by varying the playing frequency, control of the upstream coupling with a fixed lip setting allows a transition from a dominant inward (or upward) to a dominant outward striking behaviour. Near $450 \mathrm{~Hz}$, the high amplitude of $G$ provides support to lip oscillations, allowing sound to be produced despite the low value of $|Z|$. Above $450 \mathrm{~Hz}$ and below $420 \mathrm{~Hz}$, oscillations are only possible for higher values of $|Z|$, closer to a downstream impedance peak, most probably as a consequence of the lower magnitude of $G$ in this frequency range.

\section{Conclusions}

In this study, we developed an experimental method for in-vivo simulation of a vocal-tract resonance on an artificial player system. This approach allows the influence of amplitude and phase characteristics of an upstream resonance to be explored independently from other playing parameters. It reveals an optimal phase tuning of the vocal-tract at $f_{0}$ with respect to the acoustic energy produced at the input of the instrument. It also illustrates the ability of the vocal-tract to displace the playing frequency while lip and excitation 
parameters are maintained constant.

Numerical simulations involving a simple outward striking and two-dimensional lip models, along with a disturbing upstream signal representing the effect of an upstream resonance, agree with in vitro observations: variations of the phase of an upstream resonance relative to the downstream impedance at $f_{0}$ induces variations in a playing frequency, and a maximum of downstream energy occurs for a particular phase tuning of the vocal-tract impedance at $f_{0}$.

Furthermore the linearization of the flow equation around the quasi-static mouth pressure, leading to a phase condition of regeneration, qualitatively explains the displacement of the playing frequency close to a resonance of the lips when the phase of the upstream resonance reaches an appropriate value. The motion of the lips is then emphasized and the acoustic flow generated in the instrument maximized.

Overall, the effect of the growing value of $|G|$ at $f_{0}$, induced by the upstream coupling which tends to displace the playing frequency close to the lip resonance frequency, maximizes lip motion and lip opening area. It results in boosting the amplitude of the acoustic pressure created in the mouthpiece, hence contributing to an optimal efficiency of the sound production process. In some circumstances this effect may be accompanied by a growth of $|Z d|$ at $f_{0}$ leading to an increase in downstream feedback which participates in the increase in downstream pressure. This is observed for instance for the one-dimensional lip model in Fig. 9 and 8 where the decrease in playing frequency displaces oscillations closer to both the lip resonance frequency and the closest downstream impedance maxima.

While no regime change is observed with the artificial lips, numerical simulations show a regime transition induced by variations of the phase of the upstream resonance. In the case of the two-dimensional lip model, this regime change is accompanied by a switch from an outward to inward mechanism of lip oscillations.

From a music performance perspective these results highlights the potential influence of vocal-tract tuning and importance of the phase of $Z_{u}$ in controlling the playing frequency, possibly assisting regime changes, as well as optimizing the sound production process. These results are in agreement with previous in-vivo observations revealing an important influence of the vocal-tract in hte high register and at certain tone transitions [4].

From the experimental perspective, the active control method developed on the artificial players opens promising perspectives regarding the experimental investigation of the artificial lips (identification of lip resonance during performance for instance). This approach may also provide useful implications for the 
control of the artificial player and numerical models in a musical context, especially in the higher register where the downstream feedback is relatively weak and sound production is sometimes difficult to stabilize.

\section{Acknowledgement}

The authors would like to thank the Centre of Interdisciplinary Research in Music Media and Technology, the Schulich School of Music and the Natural Sciences and Engineering Research Council of Canada for helping fund this project.

\section{References}

[1] J. Wolfe, A. Z. Tarnopolsky, N. H. Fletcher, L. C. L. Hollenberg, J. Smith: Some effects of the player's vocal tract and tongue on wind instrument sound. Proc. Stockholm Music Acoustics Conference 2003, 2003, 307-310.

[2] T. Kaburagi, N. Yamada, T. Fukui, E. Minamiya: A methodological and preliminary study on the acoustic effect of a trumpet player's vocal tract. Journal of the Acoustical Society of America 130 (2011) 536-545.

[3] J. M. Chen, J. Smith, J. Wolfe: Do trumpet players tune resonances of the vocal tract? Journal of the Acoustical Society of America 131 (2012) 722-727.

[4] V. Fréour, G. P. Scavone: Acoustical interaction between vibrating lips, downstream air column, and upstream airways in trombone performance. Journal of the Acoustical Society of America 134 (2013) $3887-3898$.

[5] J. Backus: The effect of player's vocal tract on woodwind instrument tone. Journal of the Acoustical Society of America 78 (1985) 17-20.

[6] T. Idogawa, T. Kobata, K. Komuro, M. Iwaki: Nonlinear vibrations in the air column of a clarinet artificially blown. Journal of the Acoustical Society of America 93 (1993) 540-551.

[7] A. Mayer: Riam (reed instrument artificial mouth) a computer controlled excitation device for reed instruments. Proc. Stockholm Music Acoustics Conference 2003, 2003. 
[8] C. Fritz: La clarinette et le clarinettiste : influence du conduit vocal sur la production du son. PhD. Dissertation. Université Paris 6, Paris, France, 2004.

[9] D. Ferrand, T. Hélie, C. Vergez, B. Véricel, R. Caussé: Bouche artificielles asservies: étude de nouveaux outils pour l'analyse du fonctionnement des instruments á vent. Proc. 10ème Congrès Français d'Acoustique, Lyon, France, 2010.

[10] J. Gilbert, S. Ponthus, J. F. Petiot: Artificial buzzing lips and brass instruments: Experimental results. Journal of the Acoustical Society of America 104 (1998) 1627-1632.

[11] C. Vergez: Trompette et trompettiste : un systeme dynamique non lineaire a analyser, modeliser et simuler dans un contexte musical. PhD. Dissertation. Université Paris 6, Paris, France, 2000.

[12] O. Richards: Investigation of the lip reed using computational modelling and experimental studies with an artificial mouth. PhD. Dissertation. University of Edinburgh, Edinburgh, United Kingdom, 2003.

[13] T. Hélie, N. Lopes, R. Caussé: Open-loop control of a robotized artificial mouth for brass instruments. Journal of the Acoustical Society of America 131 (2012) 3470.

[14] N. Lopes, T. Hélie, R. Caussé: Control of an artificial mouth playing a trombone and analysis of sound descriptors of experimental data. Proc. Stockholm Music Acoustics Conference 2013, 2003, 521-528.

[15] C. Fritz, J. Wolfe, J. Kergomard, R. Caussé: Playing frequency shift due to the interaction between the vocal tract of the musician and the clarinet. Proc. Stockholm Music Acoustics Conference 2003, 2003, 263-266.

[16] C. Fritz, R. Caussé, J. Kergomard, J. Wolfe: Experimental study of the influence of the clarinettist's vocal tract. Proc. Forum Acusticum 2005, Budapest, Hungary, 2005.

[17] F. C. Chen, G. Weinreich: Nature of the lip reed. Journal of the Acoustical Society of America 99 (1996) 1227-1233.

[18] R. Auvray, B. Fabre, P.-Y. Lagée, S. Terrien, C. Vergez: Influence of the fluctuations of the control pressure on the sound production in flute-like instruments. Proc. Acoustics 2012 Nantes Conference, 2012, 1634-1638. 
[19] S. D. Sommerfeldt, W. J. Strong: Simulation of a player-clarinet system. Journal of the Acoustical Society of America 83 (1988) 1908-1918.

[20] G. P. Scavone: Modeling vocal-tract influence in reed wind instruments. Proc. Stockholm Music Acoustics Conference 2003, 2003, 291-294.

[21] P. Guillemain: Some roles of the vocal tract in clarinet breath attacks: Natural sounds analysis and model-based synthesis. Journal of the Acoustical Society of America 121 (2007) 2396-2406.

[22] S. Adachi, M. A. Sato: Trumpet sound simulation using a two-dimensional lip vibration model. Journal of the Acoustical Society of America 99 (1996) 1200-1209.

[23] C. Vergez, X. Rodet: Comparison of real trumpet playing, latex model of lips and computer model. Proc. International Computer Music Conference 1997, 1997.

[24] S. Bromage, M. Campbell, J. Gilbert: Open areas of vibrating lips in trombone playing. Acta Acustica united with Acustica 96 (2010) 603-613.

[25] M. Puckette, T. Apel, D. D. Zicarelli: Real-time audio analysis tools for Pd and MSP. Proc. International Computer Music Conference 1998, 1998.

[26] G. Widholm, H. Pichler, T. Ossmann: Bias, a computer-aided test system for brass wind instruments. Proc. $87^{\text {th }}$ AES Convention, 1989.

[27] N. H. Fletcher: Autonomous vibration of simple pressure-controlled valve in gas flows. Journal of the Acoustical Society of America 93 (1993) 2172-2180.

[28] J. S. Cullen, J. Gilbert, D. M. Campbell: Brass instruments: Linear stability analysis and experiments with an artificial mouth. Acta Acustica 86 (2000) 704-724.

[29] S. J. Elliot, J. M. Bowsher: Regeneration in brass instruments. Journal of Sound and Vibration 83 (1982) 181-217.

[30] S. Yoshikawa, Y. Muto: Lip-wave generation in horn players and the estimation of lip-tissue elasticity. Acta Acustica united with Acustica 89 (2003) 145-162.

[31] D. C. Copley, W. J. Strong: A stroboscopic study of lip vibrations in a trombone. Journal of the Acoustical Society of America 99 (1996) 1219-1226. 
[32] S. Adachi, M. A. Sato: Time-domain simulation of sound production in the brass instrument. Journal of the Acoustical Society of America 97 (1995) 3850-3861.

[33] C. Vergez, X. Rodet: Trumpet and trumpet player: Model and simulation in a musical context. Proc. International Computer Music Conference 2001, 2001. 